\title{
AVALIAÇÃO DA PERFORMANCE REPRODUTIVA DE FÊMEAS SELVAGENS DO CAMARÃO- ROSA FARFANTEPENAEUS BRASILIENSIS (CRUSTÁCEA: DECAPODA) EM LABORATÓRIO
}

\author{
DIOGO LUIZ DE ALCANTARA LOPES ${ }^{1}$; EDUARDO LUIS CUPERTINO BALLESTER ${ }^{1}$; WILSON WASIELESKY JUNIOR ${ }^{1}$; SÍLVIO RICARDO \\ MAURANO PEIXOTO \\ 1 - Universidade Federal do Rio Grande, FURG, Departamento de Oceanografia, Laboratório de Maricultura, CP 474, CEP 96201-900, Rio \\ Grande, RS, Brasil. E-mail: diogolalzoo@hotmail.com. Autor para correspondência. \\ 2 - Universidade Federal do Rio Grande, FURG, Departamento de Oceanografia, Laboratório de Maricultura. Endereço atual: Universidade \\ Federal Rural de Pernambuco, UFRPE, Departamento de Pesca e Aqüicultura. Campus Dois Irmãos, Recife, PE, Brasil.
}

\section{RESUMO}

A avaliação do desempenho reprodutivo do camarão Farfantepenaeus brasiliensis foi realizada na tentativa de encontrar uma espécie nativa com potencial comercial de produção. Fêmeas selvagens de F.brasiliensis $(56,89 \pm 12,36 \mathrm{~g})$ foram mantidas em sistemas de maturação convencional sem a presença de machos. Após a individualização realizada através de etiquetas numeradas coladas no dorso da carapaça e de marcações realizadas nos urópodes, 14 fêmeas foram submetidas aos procedimentos de ablação unilateral do pedúnculo ocular. O número de desovas por fêmea foi de 2,57 , com uma produção média de $71,08 \pm 41,26\left(\times 10^{3}\right)$ ovos por desova e de $187,86 \pm 131,53\left(\times 10^{3}\right)$ ovos produzidos por fêmea, perfazendo um total de $2,63\left(\times 10^{6}\right)$ ovos produzidos. A percentagem de fêmeas que apresentaram desovas fertilizadas foi de $73 \%$, com uma taxa de fertilização dos ovos de $68,42 \pm 42,2(\%)$, taxa de eclosão dos ovos de $75,92 \pm 28,90(\%)$, e taxa de metamorfose de náuplio para protozoea de $86,11 \pm 32,98(\%)$. As fêmeas selvagens de $F$. brasiliensis demonstraram respostas similares ao processo de indução a reprodução em laboratório utilizado para fêmeas de $F$. paulensis, demonstrado assim a viabilidade da utilização desta espécie como alternativa para o setor da carcinocultura brasileira.

PALAVRAS-CHAVE: Reprodução; camarão-rosa; Farfantepenaeus brasiliensis; performance reprodutiva.

\section{ABSTRACT \\ MATURATION OF THE WILD FEMALES RED-SHRIMP FARFANTEPENAEUS BRASILIENSIS (CRUSTÁCEA: DECAPODA) IN LABOTATORY}

Farfantepenaeus brasiliensis wild females $(56.89 \pm 12.36 \mathrm{~g})$ were captured and kept in a conventional unisex maturation system Reproductive performance was assessed to evaluate this species potential for commercial production. Fourteen females were individually identified by plastic labels and uropod marks, following, by eye stalk ablation was carried out in. The number of spawns per female was 2.57 with a mean production of $71.08 \pm 41.26\left(\times 10^{3}\right)$ eggs per spawn and $187.86 \pm 131.53\left(\times 10^{3}\right)$ eggs per female. Total number of eggs produced was $2.63\left(\times 10^{6}\right)$. Fertilization rate was $68.42 \pm 42.2 \%$, with $73 \%$ of the females presenting fertilized spawns. The nauplius to zoea metamorphosis rate was $86.11 \pm 32.98(\%)$. Wild $F$. brasiliensis females have shown similar results to $F$. paulensis females, demonstrating that this species has great potential for Brazilian commercial shrimp culture.

KEY WORDS: Maturation; Pink shrimp; Farfantepenaeus brasiliensis; reproductive performance.

\section{INTRODUÇÃO}

Os camarões peneídeos são um dos principais recursos pesqueiros explorados na costa Brasileira, porém um declínio na captura destes organismos vem sendo observado nos últimos 20 anos devido à sobrepesca (Reis \& D'Incao, 2000 e D'Incao \& Reis, 2002). Os camarões marinhos mais utilizados na aquacultura pertencem à família Peneidae, e estão divididos em seis gêneros de acordo com a ultima modificação taxonômica proposta por Pérez-Farfante \& Kensley (1997). Na América é observada a presença dos gêneros Farfantepenaeus e Litopenaeus (Arredondo-Figueroa, 2002).

As espécies nativas do Brasil, Farfantepenaeus brasiliensis (LATREILLE, 1817) e Farfantepenaeus paulensis (PÉREZ-FARFANTE, 1967), são conjuntamente chamados de camarão-rosa e apresentam uma sobreposição em sua distribuição na costa brasileira e, em geral, não ocorre diferenciação entre estas espécies em avaliações de estoques pesqueiros a partir de desembarques (D'Incao, 1991, Albertoni et al. 2003).

O F. brasiliensis distribui-se desde a Carolina do Norte (EUA) até a costa norte do Rio Grande do Sul (Brasil) (D'Incao, 1999). Sua reprodução ocorre na plataforma continental em profundidades entre 40 e 100 metros, os ovos são bentônicos e após a eclosão, seguem-se três etapas larvais planctônicas (nauplios, zoea e misis), cada uma com vários subestágios (Boff \& Marchiori, 1984; D'Incao, 1991). As fêmeas Farfantepenaeus apresentam télico do tipo fechado, composto por placas laterais que formam um receptáculo onde o espermatóforo é depositado no momento da cópula. A cópula ocorre no período noturno em fêmeas que recém sofreram a ecdise (muda) e o espermatóforo transferido para a fêmea pode ser utilizado para várias desovas até a próxima muda, quando é eliminado juntamente com a carapaça (Primavera, 1981; Brisson, 1986; Dall et al. 1990). 
A utilização de espécies nativas de camarões na aquacultura apresenta diversas vantagens, tais como, melhor tolerância e crescimento em baixas temperaturas, disponibilidade de reprodutores na região costeira e melhor aceitação no mercado local (Sandifer et al. 1993). Pesquisa com espécies nativas (F. paulensis) vem sendo desenvolvidas a mais de 10 anos permitindo assim, o controle da reprodução e larvicultura em laboratório e a produção em fazendas e em sistemas alternativos (Marchiores \& Boff 1983, Nakayama et al. 2009)

Durante as capturas de reprodutores selvagens de F. paulensis (litoral de Santa Catarina, Brasil) observou-se que camarões da espécie $F$. brasiliensis capturados simultaneamente apresentam algumas características desejáveis à utilização na aquacultura. Dentre estas características podemos ressaltar: A predominância durante a captura dos reprodutores selvagens de camarões $F$. brasiliensis com tamanho superior ao de F. paulensis. Maior rusticidade e resistência ao manejo de captura permitindo que os reprodutores $F$. brasiliensis cheguem ao convés da embarcação em melhores condições que $F$. paulensis, possibilitando a aquisição de reprodutores em melhores condições físicas, o que acarretará em melhor desempenho reprodutivo (Cavalli, com. pess.).

Estudos sobre a reprodução e produção em cativeiro de $F$. brasiliensis são limitados ao comportamento de cópula (Brisson 1986); indução do desenvolvimento gonadal através da ablação unilateral do pedúnculo ocular das fêmeas (Martino 1981); avaliação dos estágios ovarianos (Quintero \& Garcia, 1998). Chagas-Soares \& Pereira (1991) e Lopes (2007) realizaram desovas de fêmeas em cativeiro. Além disso, Lopes (2007) e Lopes et al. 2009, analisaram o crescimento de juvenis em sistemas alternativos de produção.

Portanto, o objetivo do presente estudo foi induzir a maturação, realizar a desova e avaliar do desempenho reprodutivo de fêmeas selvagens de Farfantepenaeus brasiliensis, na tentativa de determinar o potencial desta espécie nativa à utilização em sistemas comerciais de produção.

\section{MATERIAL E MÉTODOS}

Fêmeas selvagens de $F$. brasiliensis foram capturadas no litoral de Santa Catarina em profundidades de 35 a $45 \mathrm{~m}$ e transportadas por via rodoviária a Estação marinha de Aqüicultura. $O$ período de aclimatação aos fatores abióticos indutores a maturação (temperatura $\left( \pm 27^{\circ} \mathrm{C}\right)$, fotoperíodo (14 horas luz por dia), salinidade $( \pm 35 \mathrm{ppt}$ ) e manejo alimentar) teve duração de 7 dias. Após a aclimatação, quatorze fêmeas foram identificadas através de etiquetas numeradas fixadas na região dorsal da carapaça e marcações no urópode. As fêmeas foram divididas em dois tanques circulares de 1,50 metros de diâmetro e volume útil de 1000 litros. A indução hormonal foi realizada através da ablação unilateral do pedúnculo ocular.

A alimentação foi fornecida ad libitum, em 4 refeições diárias às 9:00, 12:00, 15:00 e 18:00 horas, tendo como itens alimentares lula (Illex sp.), mexilhão (Perna perna), músculo de peixe (Macrodon ancylodon) e ração comercial específica para reprodutores de camarões peneídeos (Breed S Inve ${ }^{\circledR}$-), conforme os procedimentos adotados para a indução a maturação de F. paulensis (Peixoto et al. 2005).

Diariamente as fêmeas maduras prestes a desovar foram selecionadas através da visualização do desenvolvimento das gônadas com o auxílio de uma lanterna (Peixoto et al. 2003a), sendo posteriormente transferidas para tanques cilíndricos individuais, com 150 litros de água marinha aquecida na mesma temperatura dos tanques de maturação, para a realização da desova. Após aproximadamente 15 horas as fêmeas selecionadas foram devolvidas aos respectivos tanques de maturação. Três amostras de $100 \mathrm{~mL}$ da água dos tanques de desovas foram coletadas após homogeneização e analisadas em microscópico ótico para determinar a ocorrência de desovas e a taxa de fertilização. Para estimar o número de ovos, três amostras de $250 \mathrm{~mL}$ de água dos tanques de desova foram retiradas após homogeneização.

Para determinar a taxa de eclosão e taxa de metamorfose para protozoea de cada desova, três amostras contendo 100 ovos e 100 náuplios foram coletadas e mantidas separadamente em béqueres de $1 \mathrm{~L}$, em sistema "banho-maria" com temperatura controlada em $26^{\circ} \mathrm{C}( \pm 1)$ e aeração suave. Para se estimar a taxa de eclosão, foi verificado o número de náuplios e ovos em cada recipiente 24 horas após a 
coleta dos ovos. Para se estimar a taxa de metamorfose para protozoea, foi verificado o número de larvas protozoea e náuplios em cada recipiente 36 horas após a coleta dos náuplios.

O potencial de utilização de fêmeas $F$. brasiliensis para reprodução em laboratório sem a presença de machos foi avaliado levando-se em consideração os seguintes aspectos: Percentagem de fêmeas selvagens fecundadas coletadas do ambiente; taxas de fertilização dos ovos, taxa de eclosão dos nauplios e de metamorfose para protozoea; intervalo em dias entre a ablação e primeira desova, entre desovas consecutivas e entre a ablação e a muda; número de desovas por fêmeas; ovos por desova; número de ovos por fêmeas; total de ovos produzidos por desova.

Para a obtenção dos valores das taxas de fertilização, eclosão e metamorfose foi levado em consideração apenas as fêmeas que não haviam realizado a muda, uma vez que a cópula nos tanques de maturação foi impossibilitada (tanques sem a presença de machos). Para a obtenção do número de ovos por desovas, foram avaliados todos os ovos produzidos independentemente da fertilização. Após a completa eclosão dos náuplios, as larvas foram transferidas para o setor de larvicultura.

As análises da qualidade da água (parâmetros físicos e químicos) foram mensuradas utilizando equipamentos com precisão de 1,0\%o para salinidade, $0,1^{\circ} \mathrm{C}$ para temperatura, 0,01 para $\mathrm{pH}$ e $0,01 \mathrm{mg}$ para oxigênio dissolvido.

\section{RESULTADOS}

\section{Qualidade da água}

Os valores (média $\pm \mathrm{DP}$ ) dos parâmetros de qualidade de água registrados durante 0 experimento foram: temperatura $(25,95 \pm 1,42)$, salinidade $(32,37 \pm 0,91), \mathrm{pH}(7,68 \pm 0,16)$ e oxigênio dissolvido $(7,08 \pm 0,74)$.

\section{Performance reprodutiva}

Os valores registrados para Intervalo entre a ablação e $1^{\circ}$ desova; Intervalo entre desovas consecutivas; Intervalo entre a ablação e a muda estão apresentados na Tabela I. O número de fêmeas utilizadas para a indução à maturação, percentagem de fêmeas fecundadas, taxa de fertilidade, eclosão e metamorfose, número total de desovas, número de ovos por desovas, número de ovos por fêmea e total de ovos produzidos, quantidade de ovos, náuplios e protozoea produzidos na primeira desova, segunda desova e terceira desova estão demonstrado na Tabela II. As fêmeas selvagens $F$. brasiliensis utilizadas em laboratório apresentaram média do peso corporal de $56,89 \pm 12,36 \mathrm{~g}$, comprimento total do corpo de $16,97 \pm 2,31 \mathrm{~cm}$ e comprimento de carapaça de 4,64 $\pm 0,60 \mathrm{~cm}$.

\section{DISCUSSÃO}

Para se obter bons resultados no processo de maturação de camarões em laboratório três aspectos básicos devem ser considerados: Indução hormonal através da ablação unilateral do pedúnculo ocular, controle dos aspectos ambientais (salinidade marinha, temperatura $27^{\circ} \mathrm{C}$, fotoperiodo 12 horas luz) e a utilização de alimentos que supram as exigências nutricionais dos reprodutores (Harrison, 1990, Browdy 1992 e Marchiori, 1996). Há um consenso entre diversos autores que o processo de ablação unilateral do pedúnculo ocular é fundamental para a indução hormonal de fêmeas de Peneideos. O F. brasiliensis demonstrou ter respostas similares aos camarões Peneideos, bem como apresentou boa respostas aos procedimentos de indução reprodutiva utilizados para o F. paulensis (Soares \& Pereira 1991; Marchiori 1996 e Peixoto et al. 2003c).

O tipo de estrutura reprodutiva feminina do Farfantepenaeus (télico fechado) permite que fêmeas selvagens e copuladas na natureza sejam utilizadas obtendo bons resultados reprodutivos em laboratório, uma vez que o espermatóforo introduzido no momento da cópula será utilizado durante as consecutivas desovas realizadas no período intermuda. A percentagem de fêmeas selvagens coletadas e fertilizadas no ambiente natural $(71,43 \%)$ demonstrou o potencial de utilização desta espécie para a reprodução em cativeiro sem a necessidade de realização de cópula em laboratório.

O desequilíbrio hormonal ocasionado pela ablação unilateral do pedúnculo ocular das fêmeas, local onde encontra-se a glândula sintetizadora do hormônio inibidor da vitelogênese, permite a 
ocorrência de desovas sucessivas em um curto período de tempo (Dall et al. 1990). Palácios et al. (1999) relataram que após 45 dias da ablação do pedúnculo ocular, as fêmeas de Litopenaeus vannamei utilizadas na reprodução em laboratório apresentaram exaustão reprodutiva, com diminuição tanto na qualidade, quanto na quantidade de ovos produzidos, tornando inviável a utilização destas fêmeas para reprodução. As fêmeas $F$. brasiliensis apresentem o mesmo comportamento de exaustão reprodutivo que o $L$. vannamei, uma vez que foi observada a diminuição do número de protozoea produzida nas desovas sucessivas (tabela II).

Diversos autores recomendam que o peso das fêmeas selvagens do gênero Farfantepenaeus utilizadas para a reprodução em laboratório esteja entre 30 e $60 \mathrm{~g}$ para se obter um bom desempenho reprodutivo (Marchiori \& Boff 1983, Peixoto et al. 2004). Neste estudo as fêmeas utilizadas apresentavam peso médio de $56,89 \pm 13,35 \mathrm{~g}$, valor este muito próximo do limite superior recomendado para as fêmeas deste gênero. $O$ maior porte e peso elevado das fêmeas afetam diretamente o período intermuda (quanto maior o reprodutor, maior será o período entre mudas), bem como animais de maior porte apresentam maior proximidade com a fase de senecência (Dall et al.1990, Bray \& Lawrence 1992). O F. brasiliensis apresentou um período médio de intermuda de 18,9 dias $( \pm 13,6)$, período este semelhante ao encontrado por Peixoto et al (2003c) para o camarão $F$. paulensis (17 dias).

Os intervalos de tempo entre a ablação do pedúnculo ocular e a primeira desova e entre desovas consecutivas estão relacionados com a eficiência dos processos fisiológicos necessários para maturação ovariana e realização da desova (Cavalli et al. 1998; Menasveta et al.,1994, Peixoto et al. 2005). Neste

\section{REFERÊNCIAS}

ALBERTONI, E.F., C. PALMA-SILVA \& F.A. ESTEVES. 2003. Overlap of Dietary Niche and Electivity of Three Shrimp Species (Crustacea: Decapoda) in a Tropical Coastal Lagoon (Rio de Janeiro, Brazil). Revista Brasileira de Zoologia, 20(1): 135-140.

ARREDONDO - FIGUEROA, J., L, 2002. El cultivo de camarón en Mexico, actualidades y perspectivas. Contactos v43, 41-54p.

BOFF, M. H. \& M.A. MARCHIORI. 1984. The effect of temperature estudo os valores observados foram de 6,69 e 8,53 dias, respectivamente sendo semelhantes aos resultados encontrados por Nakayama (2006) para $F$. paulensis $(5,3$ e 8,75).

Estudos de maturação realizados com o $F$. paulensis apresentaram taxas de fertilidade dos ovos inferior a $65 \%$, com produção de 100.000 a 160.000 ovos por desova (Cavalli et al. (1997, 1998); Peixoto et al., 2002). Embora a média do número de ovos por desova (71.080) encontrada neste estudo tenha sido inferior ao intervalo proposto para $F$. paulensis, a taxa de fertilidade dos ovos do $F$. brasiliensis $(68,42 \pm$ 42,2) está em conformidade com os estudos realizados por Peixoto et al. (2003b, 2005).

As taxas de eclosão e de metamorfose de náuplio para protozoea de camarões Peneideos estão diretamente relacionadas com a capacidade das fêmeas em transferir nutrientes para a prole, uma vez que as larvas utilizam o vitelo com única fonte de energia até atingirem o estágio de protozoea (Dall, 1990, Palácios et al. 1999). As taxas de eclosão e metamorfose do estádio larval nauplios para o estágio larva protozoea são condizentes ao observados por Peixoto et al. (2004) e Nakayama et al. 2008. Os resultados também sugerem que o manejo alimentar utilizado supriu as necessidades nutricionais das fêmeas de $F$. brasiliensis, possibilitando uma boa transferência dos nutrientes a sua prole, uma vez que as fêmeas do camarão-rosa $F$. brasiliensis foram capazes de produzir ovos e larvas de qualidade similar ao F. paulensis.

Os resultados do presente estudo demonstraram que o camarão-rosa $F$. brasiliensis apresenta similaridade com $\circ$ F. paulensis com relação ao seu desempenho reprodutivo, bem como pode representar uma alternativa para produção de camarões de espécies nativas da costa brasileira.

on larval development on the pink shrimp Penaeus paulensis. Atlântica, v7, 7-13p.

BRISSON, S. 1986. Observations on the courtship of Penaeus brasiliensis. Aquaculture, 53, 75-78.

BROWDY, C. 1992. A review of the reproductive biology of penaeus species: perspectives on controlled shrimp maturation systems for high quality nauplii production. World Aquaculture Society. Baton Rouge, LA USA

BRAY, W. A \& A. L. LAWRENCE. 1992. Reproduction Penaeus 
species in captivity. In: Marine Shrimp Culture: Principles and Practices (ed. by Fast, A. \& Lester, J.). Elsevier Science Publishers. The Netherlands. 93-170p.

CAVALLI, R. O., SCARDUA, M. P. \& WASIELESKY, W. J. 1997. Reproductive performance of different sized wild and pond-reared Penaeus paulensis females. Journal of the World Aquaculture Society, 28: 260-267.

CAVALLI, R. O., S. M. PEIXOTO \& W. J. WASIELESKY. 1998. Performance of Penaeus paulensis broodstock under long-term exposure to ammonia. Aquaculture Research, 29: 815-822.

CHAGAS-SOARES, F \& PEREIRA, O. M. 1991. Repovoamento da região lagunar-estuarina de Cananéia (SP) com Camarão-Rosa Penaeus brasiliensis. Informações preliminares. Congresso nacional de pesca e aqüicultura. Santos (SP), 22-26.

DALL, W., B. J.HILL, P. C. ROTHLISBERG \& D. J. STAPLES. 1990. The Biology of the Penaeidae. Advances in Marine Biology Academic Press, London, UK. 489p.

D'INCAO, F. 1991. Pesca e biologia de Penaeus paulensis na Lagoa dos Patos, RS. Atlântica, v12, 31-51p.

D'INCAO, F. 1999. Subordem DENDROBRANCHIATA (camarões marinhos). In: Buckup, L.; Bond-Buckup, G. 1999. "O Crustáceos do Rio Grande do Sul" Ed. Universidade/ UFRGS, Porto Alegre. 275-299p.

D'INCAO, F., \& E.G. REIS. 2002. Community-based management and technical advice in Patos Lagoon estuary (Brazil). Ocean \& Coastal Management, 45: 531-539.

HARRISON, K. E. (1990). The role of nutrition in maturation, reproduction and embryonic development of decapod crustaceans: review. Journal of shellfish Research, 9 1-28.

LOPES, D. L. A. 2007. Criação do camarão-rosa Farfantepenaeus brasiliensis (CRUSTACEA: DECAPODA) em gaiolas no estuário da Lagoa dos Patos, RS. Dissertação de mestrado. Fundação Universidade Federal de Rio Grande, Rio Grande, RS. 50p.

LOPES, D. L. A., W. J. WASIELESKY, S. PEIXOTO, E. C. BALLESTER. 2009. Análise comparativa do cultivo dos camarõesrosa Farfantepenaeus brasiliensis e Farfantepenaeus paulensis criados em gaiolas em ambiente estuarino. Ciência rural. V. 39 nº 4 (prelo).

MARCHIORI, M. A. \& M. H. BOFF. 1983. Induced maturation, spawning and larvae culture of the pink shrimp Penaeus paulensis Pérez-Farfante. Mem. - Asoc. Latinoam. Acuicult. 5, 331- 337.

MARCHIORI, A. M. 1996. Guia ilustrado de maturação e larvicultura do camarão-rosa Penaeus paulensis Pérez-Farfante, 1967. Editora FURG. Rio Grande-RS. 79p.

MARTINO, R. C. 1981. Indução a maturação em Penaeus (Farfantepenaeus) paulensis e Penaeus (Farfantepenaeus) brasiliensis através da ablação do pedúnculo ocular. Comunicado Técnico. Empresa de Pesquisa Agropecuária do Estado do Rio de Janeiro.

MENASVETA, P., S. SANGPRADUB, S. PIYATIRATITIVORAKUL \& A. W. FAST. 1994. Effects of broodstock size and source on ovarian maturation and spawning of Penaeus monodon Fabricius from the Gulf of Thailand. Journal of the World Aquaculture Society, 25: $41-49$
NAKAYAMA, C. L., WASIELESKY W. JR CAVALLI R. O. 2009. Avaliação do desempenho reprodutivo do camarão-rosa Farfantepenaeus paulensis (PÉREZ-FARFANTE, 1967) em tanques com diferentes profundidades de água. Boletim Instituto de Pesca. No prelo.

NAKAYAMA C. L., PEIXOTO .S BIANCHINI, A.; ROBALDO, R. B. \& CAVALLI R. O. 2008. Performance of Farfantepenaeus paulensis (Pe'rez-Farfante, 1967) broodstock in tanks with sand and hard substrate. Aquaculture Research, 2008, 39, 398-405.

NAKAYAMA C. L. 2006. Avaliação do uso de substrato de areia em tanques de maturação do camarão-rosa Farfantepenaeus paulensis (Pérez-Farfante, 1967). Dissertação de mestrado. Fundação Universidade Federal de Rio Grande, Rio Grande, RS. 38p.

PALÁCIOS, E., PEREZ-ROSTRO, C. I., RAMIRES, J. L., IBARRA, A. M., RACOTTA,I. S. 1999. Reproductive exhaustion in shrimp (Penaeus vanamei) reflected in larval biochemical composition, survival and growth. Aquaculture 171: 309-321.

PEIXOTO, S., R. O. CAVALLI, F. D'INCAO, W. WASIELESKY, \& N. AGUADO. 2002. Description of reproductive performance and ovarian maturation of wild Farfantepenaeus paulensis from shallow waters in southern Brazil. Nauplius, 10: 149-153.

PEIXOTO, S., WASIELESKY, W., LOUZADA, L., 2003a. Comparative analysis of pink shrimp, Farfantepenaeus paulensis and Pacific white shrimp, Litopenaeus vannamei, culture in extreme southern Brazil. J. Appl. Aquac.14, 101-111.

PEIXOTO, S., WASIELESKY, W., D'INCAO, F., CAVALLI, R.O., 2003b. Reproductive performance of similarly-sized wild and captive Farfantepenaeus paulensis. J. World Aquac. Soc. 34, 5056.

PEIXOTO, S, CAVALLI R.O. \& WASIELESKY W. 2003c. The influence of water renewal rates on the reproductive and molting cycles of Penaeus paulensis in captivity. Brazilian Archives of Biology and Technology, 46: 281-286.

PEIXOTO, S., CAVALLI, R.O., WASIELESKY, W., D'INCAO, F., KRUMMENAUER D., MILACH A. M. 2004. Effects of age and size on reproduction performances of captive Farfantepeaneus paulensisi broodstock. Aquaculture, 238: 173-182.

PEIXOTO, S., CAVALLI, R.O., WASIELESKY, W. 2005. Recent developments on broodstock maturation and reproduction of Farfantepenaeus paulensis. Brazilian Archives of Biology and technology, 48, n6, 997-1006

PÉREZ-FARFANTE, I \& B KENSLEY. 1997. Penaeoid and sergestoid shrimps and prawns of the world. Keys and diagnoses for the families and genera. Éditions du Muséum national d'Histoire naturelle, Paris.

PRIMAVERA, J. H. \& POSADAS, R. A. 1981. Studies on the egg quality of Penaeus monodon Fabricius, based on morphology and hatching rates. Aquaculture, 22: 269-277.

QUINTERO, M. E. S., \& A. GARCIA. 1998. Stages of gonadal development in the spotted pink shrimp Penaeus brasiliensis. Journal of Crustacean Biology, 18: 680-685.

REIS, E. G., \& F. D'INCAO. 2000. The present status of artisanal fisheries of extreme Southern Brazil: an effort towards communitybased management. Ocean \& Coastal Management, 43: 585-595. 
DIOGO LUIZ DE ALCANTARA LOPES; EDUARDO LUIS CUPERTINO BALLESTER; WILSON WASIELESKY JUNIOR; SÍLVIO RICARDO MAURANO PEIXOTO

SANDIFER, P.A, HOPKINS J.S., STOKES A.D. \& BROWDY C.L. 1993. Preliminary comparisons of native Penaeus setiferus and Pacific $P$. vannamei white shrimp for pond cultures in South Carolina, USA. Journal of the World Aquaculture Society, 24: 285-303.
SOARES, F. C \& PEREIRA, O. M. 1991. Repovoamento da região lagunar- estuarina de Cananéias (SP) com camarão-rosa Penaeus brasiliensis: informe preliminares. Controle de pesca e aqüicultura 22-26 julho de 1991.

Recebido: 04/07/2007

Aceito: 19/07/2010 